\title{
Research on Displacement Transfer Characteristics of a New Vibration-Isolating Platform Based on Parallel Mechanism
}

\author{
Shi Peicheng, Shi Peilei, Nie Gaofa, Tang Ye, and Pan Daoyuan \\ College of Mechanical \& Automotive Engineering, Anhui Polytechnic University, No. 54, Beijing Middle Road, Wuhu City, China \\ Correspondence should be addressed to Shi Peicheng; shipeicheng@126.com
}

Received 15 February 2017; Accepted 18 April 2017; Published 8 June 2017

Academic Editor: Roman Lewandowski

Copyright (C) 2017 Shi Peicheng et al. This is an open access article distributed under the Creative Commons Attribution License, which permits unrestricted use, distribution, and reproduction in any medium, provided the original work is properly cited.

\begin{abstract}
Based on the parallel mechanism theory, a new vibration-isolating platform is designed and its kinetic equation is deduced. Taylor expansion is used to approximately replace the elastic restoring force expression of vibration-isolating platform, and the error analysis is carried out. The dynamic-displacement equation of the vibration-isolating platform is studied by using the Duffing equation with only the nonlinear term. The dynamic characteristics of the vibration-isolating platform are studied, including amplitude-frequency response, jumping-up and jumping-down frequency, and displacement transfer rate under base excitation. The results show that the lower the excitation amplitude, the lower the initial vibration isolation frequency of the system. The influence of the platform damping ratio $\zeta$ on displacement transfer rate is directly related to the jumping-down frequency $\Omega_{d}$ and the external excitation frequency. The vibration-isolating platform is ideally suited for high-frequency and small-amplitude vibrations.
\end{abstract}

\section{Introduction}

The existing research has proved that the parallel mechanism has the advantages of compact structure, good stiffness, and strong bearing capacity and can be applied to multidimensional vibration isolation [1-3]. Zhang et al., based on the Steward platform, realized the control of multi-degree-offreedom system and achieved multidimensional vibration control through taking the specific displacement vector as the generalized coordinates to control the active platform [4]. Based on the Steward platform, the HAVI system was produced in the American IA company, the SUITE designed by CSA was used in satellite vibration isolation, and ULB developed a novel vibration isolation system, and so forth [5]. Guangfu et al. accomplished the experiment research of vibration control of a two-degree-of-freedom system via adopting LMS adaptive algorithm and simulated the controlled six-degree-of-freedom system designed on the basis of the Steward platform. The results showed that the vibration level of controlled system is less than $10 \%$ of the original vibration [6]. Ma et al., in Jiangsu University, developed different multidimensional vibration system based on parallel mechanism [7-9].
All in all, researchers at home and abroad obtained large number of achievements related to vibration isolation system related to parallel mechanism on both the vibration response and the control strategy. But most researches of the multidimensional vibration isolation conducted by the scholars were based on the Steward platform; the vibration isolation characteristics of the non-Steward platform was rarely to be seen. The studies above were based on the active control and there were no variable stiffness and variable damping researches based on passive vibration isolation technology in the field.

Based on previous studies, this paper presents an integrated innovation and puts forward the design theory of variable damping and variable stiffness vibration-isolating platform based on parallel mechanism, which makes the platform have the operating characteristics of "large damping in lowfrequency large-amplitude movement and small damping in high-frequency small-amplitude movement" and "high static stiffness and low dynamic stiffness." Through the structural design and spatial layout of the multibar mechanism (as shown in Figure 1), instead of the external control source method, the two inherent defects of the traditional linear vibration-isolating system are solved: (1) the conflict between 


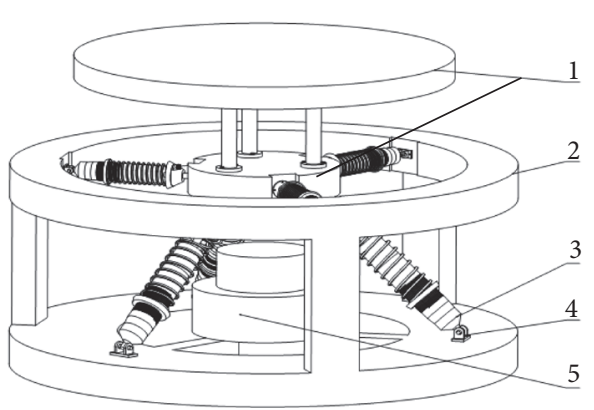

Figure 1: Schematic diagram of prototype design. In the figure: 1, bearing platform; 2 , base; 3 , damper; 4 , fixed hinge; 5 , down limit pad.

the inherent frequency and excitation frequency of vibrationisolating platform in low-frequency or ultra-low-frequency vibration isolation; (2) the conflict between the static bearing capacity and low stiffness $[2,10,11]$. At the same time, the sixdimensional vibration reduction of single-layer structure is realized. Such kind of vibration-isolating platform is expected to be widely used in many industries such as ambulance beds, automobiles, ships, aircraft, aerospace vehicles, precision instruments and meters transportation, and military equipment $[2,12]$. This paper focuses on the displacement transfer characteristics of the vibration-isolating platform.

\section{Physical Model and Kinetic Equation of the Vibration-Isolating Platform}

2.1. Physical Model of the Platform. The new vibrationisolating platform based on parallel mechanism, designed by our research team, is shown in Figure 1. In Figure 1, the upper and lower disks of the bearing platform are fixedly connected, the upper disk is used to place the target vibration isolation object, and the lower disk is used to connect the six supports (damper and its spring components). The outer periphery of damper 3 is arranged with a coil spring, and the lower base of the spring is screwed to the outer peripheral surface of damper 3 , and the base can be moved up and down along the damper axis to give the spring a different initial amount of compression. When the spring base is still unable to meet the required load capacity, the spring can be replaced by a spring with greater or smaller stiffness to achieve variable stiffness. The dampers of the damper 3 are adjustable in six positions: six dampers and three spring assemblies are arranged between the upper end face of base 2 and the bearing platform, and three of them are arranged between the lower end face and the bearing platform. The functional principle of the three arranged on the upper end face is the same as that of the lower end face, but the model is smaller. The whole platform can be damped at least in one dimension and can be damped in a maximum of six dimensions; the vertical motion is shown in Figures 2 and 3.

By adjusting the initial compression amount of the three springs in the upper end, the whole platform can have quasi-zero stiffness in the vertical direction. If the quasizero stiffness characteristic can not be pursued, the platform

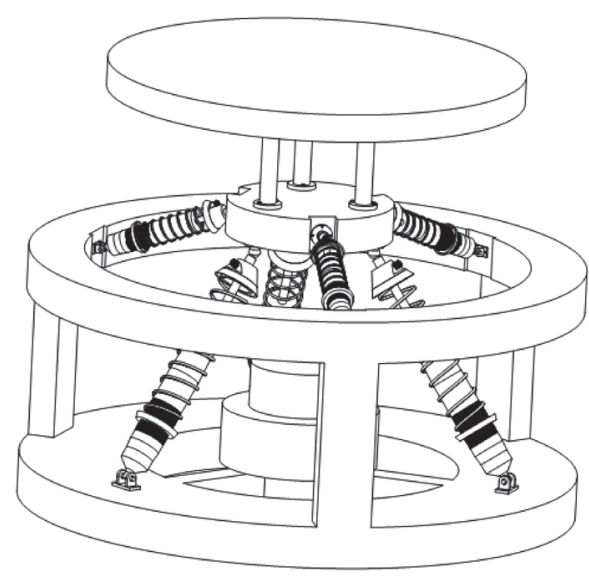

FIGURE 2: Vertical upward jump of prototype.

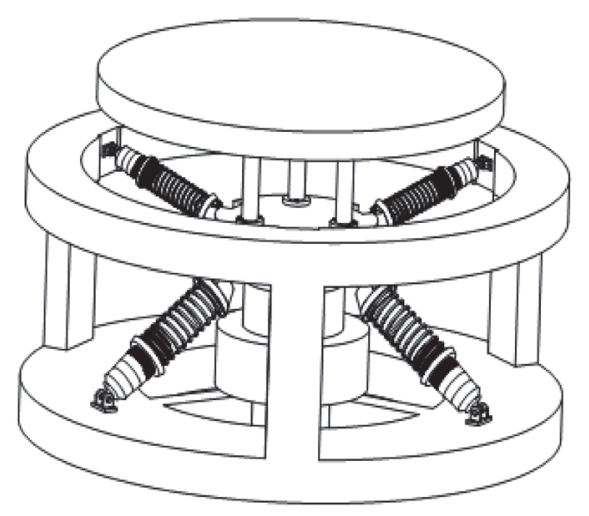

Figure 3: Vertical downward jump of prototype.

can be equated with the parallel mechanism-based vibrationisolating platform developed by Professor $\mathrm{Ma}$ et al., but it can only isolate the intermediate-frequency and highfrequency vibrations with an excitation frequency greater than $\sqrt{2}$ times of the inherent frequency of the system. The damping or damping ratio of the whole platform can be adjusted according to the external excitation frequency by the damper's adjustment handle so that the vibration-isolating platform has the best damping ratio. If the external excitation frequency is wide, synchronized adjustment of the damping and stiffness can be made to make the platform achieve the best vibration isolation match.

2.2. Static Equation of Platform. The simplified vertical mechanical model of the vibration isolation platform is shown in Figure 4. In the figure, $b$ and $a$ are the distances from the hinge centers of the upper and lower springs and the outer frame to the axis of the vibration reduction platform, respectively. The displacement $x$ of the initial position is defined as the vertical displacement of the intersection point $O$ of upper and lower springs. $H$ represents the vertical distance between $O_{a}$ and $O_{b}$, the centers of upper and lower platforms. $h$ is the vertical distance between the initial top intersection $O$ of the inclined springs and the static 


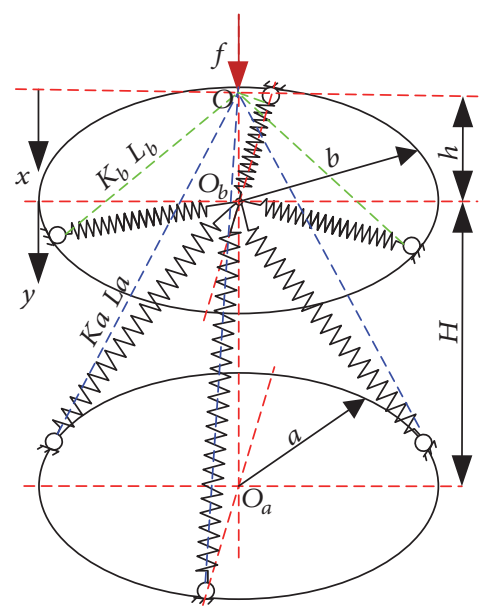

FIGURE 4: Vertical mechanical model of the vibration isolation platform.

equilibrium position, where the platform starts to have quasizero stiffness, assuming the static equilibrium position of the vibration isolation platform coincides with the center $O_{b}$. $L_{b}$ and $L_{a}$ are the original lengths of the upper and lower springs, respectively, when the top intersection point $O$ of the inclined springs is in the initial position.

As shown in Figure 4, the lower spring with the stiffness of $k_{a}$ and the upper one with the stiffness of $k_{b}$ intersect at the point $O$. Both the upper and lower springs are slightly compressed. With the vertical static force $f$ acting at the initial position $O$, the relationship between the total elastic recovery force $F$ and the displacement $x$ of the vibration isolation platform is given by

$$
F=3 k_{a}\left(L_{a}-l_{a}\right) \sin a+3 k_{b}\left(L_{b}-l_{b}\right) \sin b,
$$

where $\sin a=(H+h-x) / \sqrt{a^{2}+(H+h-x)^{2}}, \sin b=$ $(h-x) / \sqrt{b^{2}+(h-x)^{2}}, l_{a}=\sqrt{a^{2}+(H+h-x)^{2}}, l_{b}=$ $\sqrt{b^{2}+(h-x)^{2}}$, which are the working lengths of upper and lower springs, respectively, when the displacement is $x$.

So (1) can be expressed as

$$
\begin{aligned}
F= & 3 k_{a}(H+h-x)\left(1-\frac{L_{a}}{\sqrt{a^{2}+(H+h-x)^{2}}}\right) \\
& +3 k_{b}(h-x)\left(1-\frac{L_{b}}{\sqrt{b^{2}+(h-x)^{2}}}\right) .
\end{aligned}
$$

With the vertical displacement $y$ representing the displacement of the intersection point $O$ from the static

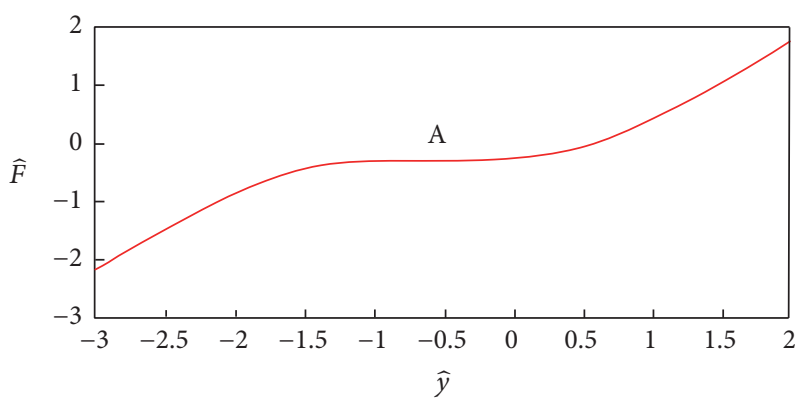

FIGURE 5: Force-displacement curve of the platform under selected parameters.

equilibrium position of the vibration isolation platform, that is, the position of $O_{b}$, then (2) can be transformed into

$$
\begin{aligned}
F= & 3 k_{a}(H+y)\left(1-\frac{L_{a}}{\sqrt{a^{2}+(H+y)^{2}}}\right) \\
& +3 k_{b} y\left(1-\frac{L_{b}}{\sqrt{b^{2}+y^{2}}}\right) .
\end{aligned}
$$

When both sides of (3) are divided by $3 k_{b} L_{b}$, the dimensionless force-displacement equation of the platform can be obtained:

$$
\begin{aligned}
\widehat{F}= & \lambda(\widehat{H}+\widehat{y})\left(1-\frac{\widehat{L}_{a}}{\sqrt{\left(\widehat{a}^{2}+(\widehat{H}+\widehat{y})^{2}\right)}}\right) \\
& +\widehat{y}\left(1-\frac{1}{\sqrt{\hat{b}^{2}+\widehat{y}^{2}}}\right),
\end{aligned}
$$

where $\hat{y}=y / L_{b}, \widehat{a}=a / L_{b}, \widehat{b}=b / L_{b}, \widehat{L}_{a}=L_{a} / L_{b}, \lambda=$ $k_{a} / k_{b}, \widehat{H}=H / L_{b}, \widehat{F}=F / 3 k_{b} L_{b}$.

If we take the derivative of $\hat{y}$ on both sides of (4), the dimensionless equation about stiffness can be expressed as follows:

$$
\begin{aligned}
\widehat{k}= & \left(1-\frac{\widehat{b}^{2}}{\left(\widehat{b}^{2}+\widehat{y}^{2}\right)^{3 / 2}}\right) \\
& +\lambda\left(1-\frac{\widehat{L}_{a} \widehat{a}^{2}}{\left(\widehat{a}^{2}+(\widehat{H}+\widehat{y})^{2}\right)^{3 / 2}}\right) .
\end{aligned}
$$

When a set of coefficients are given, the forcedisplacement curve of the vibration isolation platform can be got, as is demonstrated in Figure 5. It can be seen that the platform displays stable quasi-zero stiffness in a small range when $\hat{y}=-0.75$. 
2.3. Dynamic Equation of Platform. It is necessary to simplify (4) for the convenience of dynamic calculation and analysis. Consequently, (4) is extended at point A (shown in Figure 4); that is, $\hat{y}$ equals -0.75 , according to the definition of Taylor series. The former three orders are kept. So it can be expressed:

$$
\begin{aligned}
\widehat{F}= & \widehat{F}(-0.75)+\widehat{F}^{\prime}(-0.75) \widehat{y}+\frac{\widehat{F}^{\prime \prime}(-0.75)}{2 !} \widehat{y}^{2} \\
& +\frac{\widehat{F}^{\prime \prime \prime}(-0.75)}{3 !} \widehat{y}^{3}
\end{aligned}
$$

According to (4) and (5), the first derivative of elastic recovery force $F$ at point $\mathrm{A}$ is $\widehat{F}^{\prime}(-0.75)=\widehat{k}(-0.75)=0$, the second derivative is $\widehat{F}^{\prime \prime}(-0.75)=\widehat{k}^{\prime}(-0.75)=0$, and the third is

$$
\begin{aligned}
\widehat{F}^{\prime \prime \prime}(-0.75)= & \frac{3 b^{4}-12 b^{2} \widehat{y}^{2}}{\left(b^{2}+\widehat{y}^{2}\right)^{7 / 2}} \\
& +\frac{3 \lambda L_{a} a^{4}-12 \lambda L_{a} a^{2}(H+\widehat{y})^{2}}{\left(a^{2}+(H+\widehat{y})^{2}\right)^{7 / 2}} \\
= & \frac{3 b^{4}-6.75 b^{2}}{\left(b^{2}+0.75^{2}\right)^{7 / 2}} \\
& +\frac{3 \lambda L_{a} a^{4}-12 \lambda L_{a} a^{2}(H+0.75)^{2}}{\left(a^{2}+(H+0.75)^{2}\right)^{7 / 2}} .
\end{aligned}
$$

So the approximate elastic recovery force $F$ can be presented as

$$
\widehat{F}=\widehat{F}(-0.75)+\frac{\widehat{F}^{\prime \prime \prime}(-0.75)}{3 !} \widehat{y}^{3}
$$

If set

$$
\widehat{F}_{1}=\widehat{F}-\widehat{F}(-0.75)
$$

then

$$
\begin{aligned}
\widehat{F}_{1} & =\frac{\widehat{F}^{\prime \prime \prime}(-0.75)}{6} \widehat{y}^{3}=\frac{\widehat{y}^{3}}{6}\left(\frac{3 b^{4}-6.75 b^{2}}{\left(b^{2}+0.75^{2}\right)^{7 / 2}}\right. \\
& \left.+\frac{3 \lambda L_{a} a^{4}-12 \lambda L_{a} a^{2}(H+0.75)^{2}}{\left(a^{2}+(H+0.75)^{2}\right)^{7 / 2}}\right) .
\end{aligned}
$$

Inclusion of only the third derivative in (10) offers convenience of analysis of dynamic performance of the vibration isolation platform. The error between (10) and (4) is shown in Figure 6.

It can be seen from Figure 6 that the approximate curve largely coincides with the accurate one in the interval $(-2.4$, -1.3 ). When the intersection point of the spring vibrates near point $\mathrm{A}$ in the small range, the approximate curve coincides exactly with the accurate one, making it feasible to replace

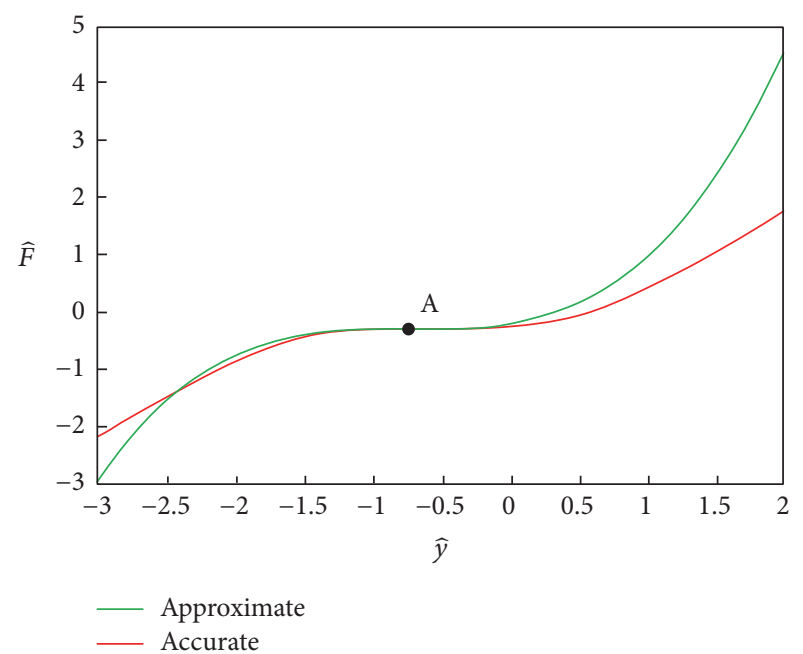

FIGURE 6: Error between approximate force-displacement curve and accurate one.

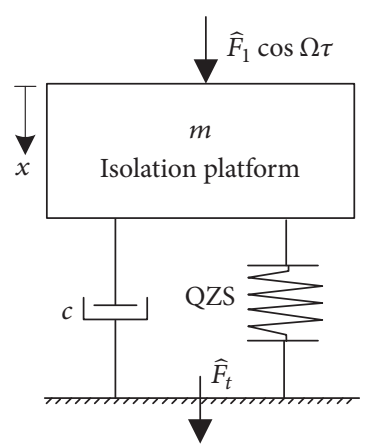

Figure 7: Dynamic model of platform.

the accurate equation (4) of elastic recovery force with the approximate equation (10) in the range of small vibration.

Supposing that the vibration isolation platform slightly vibrates in small range near point $\mathrm{A}$, where the stiffness tends to zero, the simplified dynamic model of the platform is shown in Figure 7.

Under the simple harmonic excitation condition, the approximate force-displacement equation of the platform can be presented as follows:

$$
\widehat{F}_{1}=\gamma \widehat{y}^{3}
$$

where the stiffness of the system

$$
\begin{aligned}
\gamma & =\frac{1}{6}\left(\frac{3 b^{4}-6.75 b^{2}}{\left(b^{2}+0.75^{2}\right)^{7 / 2}}\right. \\
& \left.+\frac{3 \lambda L_{a} a^{4}-12 \lambda L_{a} a^{2}(H+0.75)^{2}}{\left(a^{2}+(H+0.75)^{2}\right)^{7 / 2}}\right)
\end{aligned}
$$


Furthermore, (11) can be changed to Duffing equation that contains only nonlinear terms to represent the forcedisplacement equation of the vibration isolation platform [13-15]. It can be expressed as follows:

$$
\widehat{\ddot{x}}+2 \zeta \hat{\dot{x}}+\gamma \widehat{x}^{3}=\widehat{F} \cos \Omega \tau,
$$

where $\hat{x}=x / L_{b}, \zeta=c \omega_{0} / 2 k_{a}, \omega_{0}^{2}=k_{a} / m, \tau=\omega_{0} t, \Omega=$ $\omega / \omega_{0}, \widehat{F}=F / 3 k_{b} L_{b}$.

2.4. Amplitude-Frequency Characteristic of Platform. By using the harmonic balance method, the same order harmonic term on both sides of the differential equation has the same coefficient. In the meanwhile, only the predominant excitation frequency is considered, ignoring higher harmonic terms $[16,17]$. The periodic response of (13) is as follows:

$$
\widehat{x}(\tau)=A \sin (\Omega \tau+\theta),
$$

where $A$ is the system response amplitude, $\theta$ represents the response phase of the system, and $\Omega$ is the harmonic excitation frequency.

Upon substitution of (14) into (13), it can be got that

$$
\begin{aligned}
\left(\frac{3}{4} \gamma A^{3}-A \Omega^{2}\right) \cos \theta-2 A \zeta \Omega \sin \theta & =\widehat{F} \\
-2 A \zeta \Omega \cos \theta-\left(\frac{3}{4} \gamma A^{3}-A \Omega^{2}\right) \sin \theta & =0 .
\end{aligned}
$$

On elimination of phase $\theta$ in (15), the amplitudefrequency characteristic equation of the platform can be obtained:

$$
\left(\frac{3}{4} \gamma A^{3}-A \Omega^{2}\right)^{2}+(2 \zeta A \Omega)^{2}=\widehat{F}^{2}
$$

With the stiffness ratio $\gamma=0.13$, damping ratio $\zeta=$ 0.02 , and excitation force amplitude $\widehat{F}=0.05$, the vibration amplitude-frequency characteristic curve of the platform can be acquired by the use of command ezplot () of Matlab implicit function, as is shown in Figure 8.

As can be seen in Figure 8, when the excitation frequency changes in different directions, the system amplitude response is as follows.

(1) When the Excitation Frequency Gradually Increases from Zero. When the excitation frequency increases from zero, the response amplitude changes are shown in the green arrow line in Figure 8. The response amplitude first increases along the upper branch, namely, the resonant branch: starting from point 1 , via point 2 , and then reaching the point 3 , which corresponds to the maximum amplitude and the jumpingdown frequency $\Omega_{d}$. That is to say, as the excitation frequency continues to increase, the response amplitude jumps directly to point 4 on the lower branch, the nonresonant branch, and finally to point 5 and extends infinitely.

(2) When the Excitation Frequency Gradually Decreases from Infinity to Zero. When the excitation frequency decreases from high frequency to low frequency, the changing tendency

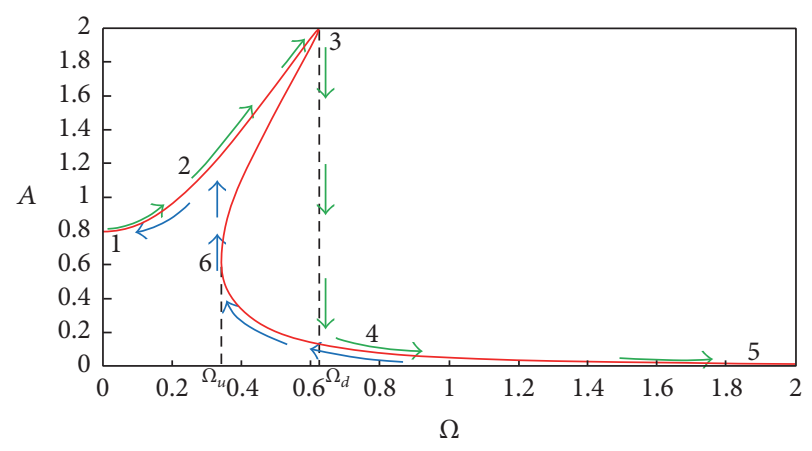

FIGURE 8: Amplitude-frequency characteristic curve of platform. $\Omega_{u}$ is jumping-up frequency and $\Omega_{d}$ is jumping-down frequency.

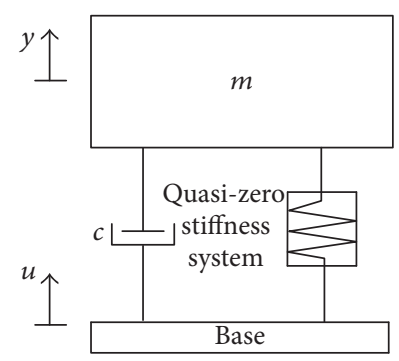

FIGURE 9: Schematic diagram of system displacement transfer rate.

of the response amplitude is shown in the blue arrow line in Figure 8. The response amplitude first increases along the nonresonant branch: starting from point 5 , via point 4 , and then reaching point 6 , which corresponds to the jumpingup frequency $\Omega_{u}$. That is to say, as the excitation frequency continues to decrease; the response amplitude jumps directly to point 2 on the resonant branch and finally changes to point 1 along the resonant branch.

In summary, only when the external excitation frequency is larger than $\Omega_{d}$ will the amplitude response of the system certainly change along the nonresonant branch. The excitation frequencies in the jumping range $\left(\Omega_{u}<\Omega<\right.$ $\Omega_{d}$ ) result in uncertain amplitude-frequency response of the system, which may fall to the resonant branch curve with large amplitude or on the nonresonant curve with small amplitude. It is obvious that only when the amplitudefrequency response changes along the nonresonant branch is the system equipped with vibration isolation characteristics.

In general, to make the solution of its dynamic differential equation tend to the nonresonant branch with small amplitude, it is required that the excitation frequency $\Omega$ be larger than the jumping-down frequency $\Omega_{d}$ for nonlinear vibration isolation system.

\section{Displacement Transfer Characteristics of the Platform}

3.1. Displacement Transfer Rate under Base Excitation. Set base displacement excitation $u=-u_{0} \cos \Omega \tau$, which is shown in Figure 9, the mass of vibration isolation object is $m$, and 
the nonlinear dynamic differential equation of the vibrationisolating platform obtained by Newton's second law is

$$
m \ddot{y}+c(\dot{y}-\dot{u})+\gamma(y-u)^{3}=0 .
$$

The relative displacement between vibration isolation object and base is $x=y-u$. Then

$$
\begin{aligned}
& x=y-u \\
& \dot{x}=\dot{y}-\dot{u} \\
& \ddot{x}=\ddot{y}-\ddot{u},
\end{aligned}
$$

and substitute in formula (17) to obtain the motion equation of displacement $x$ as follows:

$$
m \ddot{x}+c \dot{x}+\gamma x^{3}=-m \ddot{u}=m u_{0} \Omega^{2} \cos \Omega \tau .
$$

Conduct nondimensional processing on formula (19) to obtain the displacement response of vibration-isolating platform expressed by Duffing equation as follows:

$$
\ddot{\tilde{x}}+2 \zeta \dot{\hat{x}}+\gamma \widehat{x}^{3}=\widehat{f} \cos \Omega \tau,
$$

where $\hat{x}=x / L_{b}, \zeta=c \omega_{0} / 2 k_{a}, \omega_{0}^{2}=k_{a} / m, \tau=\omega_{0} t, \Omega=$ $\omega / \omega_{0}, \widehat{f}=\widehat{u}_{0} \Omega^{2}, \widehat{u}_{0}=u_{0} / L_{b}$,

$$
\begin{aligned}
\gamma & =\frac{1}{6}\left(\frac{3 b^{4}-6.75 b^{2}}{\left(b^{2}+0.75^{2}\right)^{7 / 2}}\right. \\
& \left.+\frac{3 \lambda L_{a} a^{4}-12 \lambda L_{a} a^{2}(H+0.75)^{2}}{\left(a^{2}+(H+0.75)^{2}\right)^{7 / 2}}\right) .
\end{aligned}
$$

Set the system displacement response as $\widehat{x}(\tau)=$ $\rho \cos (\Omega \tau+\phi)$; then

$$
\dot{\hat{x}}(\tau)=-\rho \Omega \sin (\Omega \tau+\phi) .
$$

By the constant variation method, $\rho$ and $\phi$ are the functions of time $\tau$; namely, $\widehat{x}(\tau)=\rho(\tau) \cos (\Omega \tau+\phi(\tau))$; then

$$
\begin{aligned}
\dot{\hat{x}}(\tau)= & -\rho(\tau) \Omega \sin (\Omega \tau+\phi(\tau)) \\
& -\rho(\tau) \dot{\phi}(\tau) \sin (\Omega \tau+\phi(\tau)) \\
& +\dot{\rho}(\tau) \cos (\Omega \tau+\phi(\tau)) .
\end{aligned}
$$

Formula (23) and formula (22) should be in the same form; there must be

$$
\begin{aligned}
& -\rho(\tau) \dot{\phi}(\tau) \sin (\Omega \tau+\phi(\tau))+\dot{\rho}(\tau) \cos (\Omega \tau+\phi(\tau)) \\
& \quad=0
\end{aligned}
$$

Therefore,

$$
\begin{aligned}
\ddot{\widehat{x}}(\tau)= & -\rho(\tau) \Omega^{2} \cos (\Omega \tau+\phi(\tau)) \\
& -\rho(\tau) \dot{\phi}(\tau) \Omega \cos (\Omega \tau+\phi(\tau)) \\
& -\dot{\rho}(\tau) \Omega \sin (\Omega \tau+\phi(\tau)) .
\end{aligned}
$$

Substitute $\dot{\hat{x}}(\tau), \ddot{\vec{x}}(\tau)$ into formula (20) and combined with formula (24) we can obtain

$$
\begin{aligned}
& \rho^{\prime}=-\frac{1}{\Omega} U \sin (\Omega \tau+\phi) \\
& \phi^{\prime}=-\frac{1}{\rho \Omega} U \cos (\Omega \tau+\phi),
\end{aligned}
$$

where $U=u_{0} \Omega^{2} \cos \Omega \tau+\rho \Omega^{2} \cos (\Omega \tau+\phi)+2 \rho \Omega \zeta \sin (\Omega \tau+$ $\phi)-\gamma \rho^{3} \cos ^{3}(\Omega \tau+\phi)$.

According to the definition of average method, the right side of formula (26) is substituted by the average value of one cycle of displacement response; we can obtain

$$
\begin{aligned}
& \rho^{\prime}=-\frac{\Omega}{2 \pi} \int_{0}^{2 \pi / \Omega} \frac{1}{\Omega} U \sin (\Omega \tau+\phi) d \tau \\
& \phi^{\prime}=-\frac{\Omega}{2 \pi} \int_{0}^{2 \pi / \Omega} \frac{1}{\rho \Omega} U \cos (\Omega \tau+\phi) d \tau .
\end{aligned}
$$

Let the left be zero; integrate the above formula:

$$
\begin{aligned}
-\frac{u_{0} \Omega^{2} \sin \phi}{2 \Omega}-\rho \zeta & =0 \\
-\frac{u_{0} \Omega^{2} \cos \phi}{2 \rho \Omega}-\frac{\Omega}{2}+\frac{3 \gamma \rho^{2}}{8 \Omega} & =0 .
\end{aligned}
$$

Add the squares of the above two formulas to obtain the amplitude-frequency response function shown in formula (29):

$$
\Omega^{4} u_{0}{ }^{2}=\left(\frac{3}{4} \gamma \rho^{3}-\rho \Omega^{2}\right)^{2}+4 \rho^{2} \Omega^{2} \zeta^{2}
$$

This expression expresses that the amplitude $\rho$ of the excitation amplitude of support after passing through the vibration-isolating platform and being transferred to the vibration isolation object is related to the damping ratio $\zeta$, stiffness ratio $\gamma$, and external excitation frequency $\Omega$ of the system. Formula (29) is regarded as the equation of $\Omega^{2}$; we can obtain

$$
\Omega_{1,2}=\frac{1}{2} \sqrt{\frac{M \pm N}{H}},
$$

where $H=\rho^{2}-u_{0}^{2}, M=3 \gamma \rho^{4}-8 \zeta^{2} \rho^{2}, N=$ $\rho^{2} \sqrt{64 \zeta^{4}-48 \gamma \zeta^{2} \rho^{2}+9 \gamma^{2} u_{0}^{2} \rho^{2}}$.

Let $\Omega_{1}=\Omega_{2}$; we can obtain the maximum response amplitude:

$$
\rho_{\max }=\frac{8 \zeta^{2}}{\sqrt{48 \gamma \zeta^{2}-9 \gamma^{2} u_{0}^{2}}} .
$$

In order to ensure that the response of the system is bounded, namely, to ensure that the resonance curve has a stable section, formula (31) shall meet

$$
\frac{16 \zeta^{2}}{3 \gamma u_{0}^{2}}>1
$$




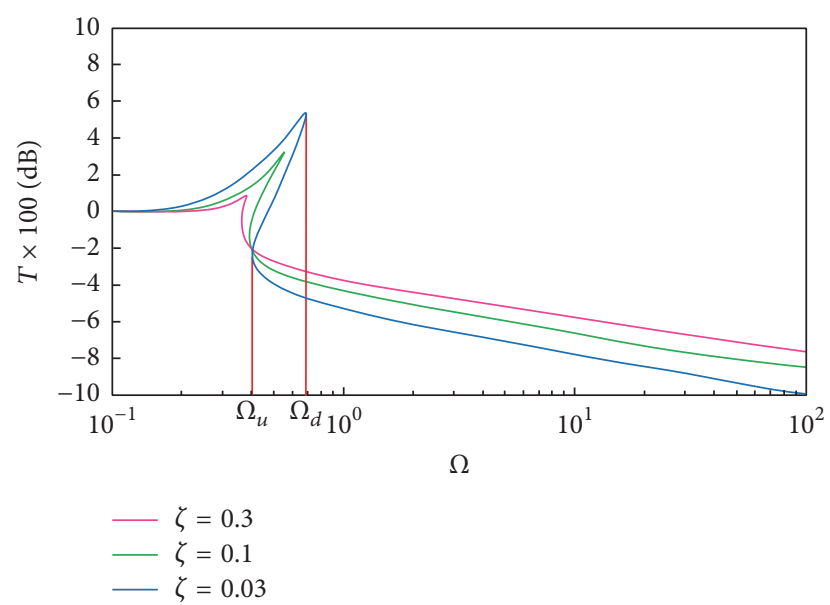

FIGURE 10: The relationship between damping ratio and displacement transfer rate.

Substitute $\rho_{\max }$ into formula (32) to obtain the jumpingdown frequency $\Omega_{d}$

$$
\Omega_{d}=\zeta^{3} \sqrt{\frac{24 \gamma u_{0}^{2}-64 \zeta^{2}}{64 \zeta^{6}-60 \gamma \zeta^{4} u_{0}^{2}+18 \gamma^{2} \zeta^{2} u_{0}^{4}-27 \gamma^{3} u_{0}^{6}}}
$$

Set the displacement response of the vibration isolation object as $Z$; then $Z=\rho+u_{0}$; namely,

$$
|Z|=\sqrt{\rho^{2}+u_{0}^{2}+2 \rho u_{0} \cos \phi} .
$$

According to the definition of displacement transfer rate, the displacement transfer rate of the platform is

$$
T=\frac{\sqrt{\rho^{2}+u_{0}^{2}+2 \rho u_{0} \cos \phi}}{u_{0}},
$$

where $\cos \phi=\rho / u_{0}+3 \gamma \rho^{3} / 4 u_{0} \Omega^{2}$.

When considering only the vertical spring in the mechanism, the mass of vibration isolation object is only carried by a vertical spring. The vibration-isolating platform becomes a traditional linear vibration-isolating platform. Through the analysis above, it is easy to get the displacement transfer rate of the linear system shown in formula (36):

$$
T_{1}=\sqrt{\frac{1+(2 \zeta \Omega)^{2}}{\left(1-\Omega^{2}\right)^{2}+(2 \zeta \Omega)^{2}}} .
$$

\subsection{The Relationship between Damping Ratio and Displace-} ment Transfer Characteristics. Let stiffness $\gamma=2$ and excitation displacement amplitude $u_{0}=0.04$. Figure 10 shows that, in the left and right frequency range with the jumping-down frequency $\Omega_{d}$ as the cut-off point, the effect of the system's damping ratio $\zeta$ on the displacement transfer rate is different. When the excitation frequency is in the high-frequency band that is higher than the jumping-down frequency $\Omega_{d}$ of the platform, the smaller the damping ratio $\zeta$, the better. When

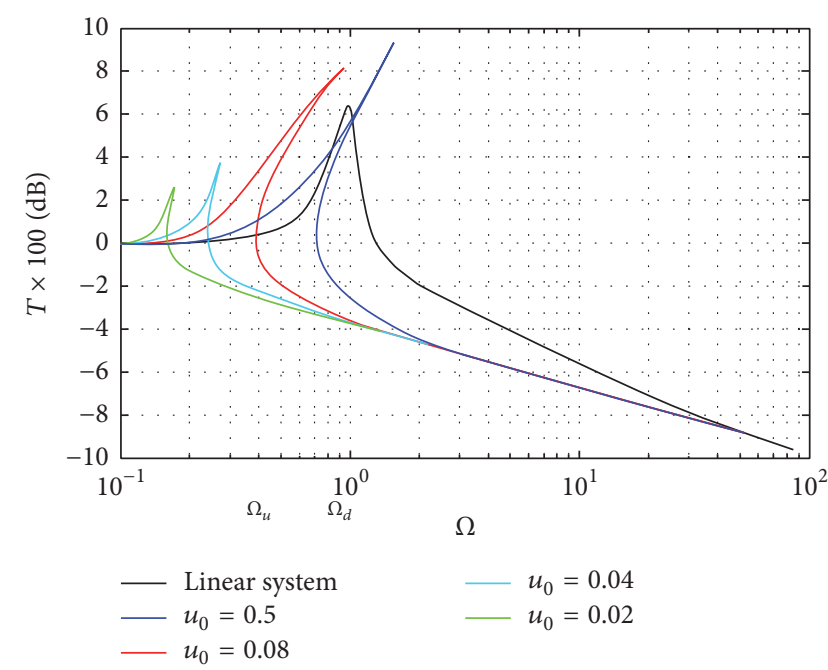

FIGURE 11: The relationship between displacement excitation amplitude and displacement transfer rate.

the excitation frequency is in the low-frequency band that is lower than the jumping-down frequency $\Omega_{d}$ of the platform, the larger the damping ratio $\zeta$, the better.

3.3. Effect of Displacement Excitation of Support on Displacement Transfer Rate. Let damping ratio $\zeta=0.02$ and stiffness $\gamma=2$. Figure 11 shows the effect of displacement excitation amplitude of support on the displacement transfer rate, and the comparison between the linear system and the vibrationisolating platform system with quasi-zero stiffness characteristic designed in this paper is made. The linear system shown in the figure refers to the remaining system after the negative-stiffness mechanism is removed from the quasi-zero stiffness vibration-isolating platform. According to Figure 11, the displacement excitation amplitude has a great influence on the vibration isolation performance of the system. When the excitation amplitude is large $\left(u_{0}=0.5\right)$, the initial vibration isolation frequency (i.e., jumping-down frequency $\Omega_{d}$ ) of the system is greater than the inherent frequency of the corresponding linear system, and the isolation effect is not as good as that of the linear system. When the excitation amplitude is decreased gradually, the initial vibration isolation frequency of the system decreases and the lowfrequency vibration isolation effect becomes apparent. This phenomenon proves that the vibration-isolating platform is suitable for small vibrations.

For different excitation amplitudes, when the external excitation frequency is large enough, the displacement transfer rate curve of the vibration isolation system and its corresponding linear system will tend to coincide, indicating that the system has the same vibration isolation capacity in the high-frequency band as in the linear vibration-isolating system. But in the low-frequency region, the vibration isolation effect of the system is obviously better than that of the corresponding linear system. With the reduction of the displacement excitation amplitude, the difference between the two becomes more and more obvious. 
With the decrease of displacement excitation amplitude $u_{0}$, the frequency range covered by the resonant branch decreases, the jumping-down frequency $\Omega_{d}$ decreases, the corresponding maximum amplitude decreases, and the jumping phenomenon eventually disappears.

\section{Conclusion}

Based on the parallel mechanism theory, a new vibrationisolating platform is designed, and the dynamic characteristics of the vibration-isolating platform are studied, including amplitude-frequency response, up and down hopping frequency, and displacement transfer rate, providing some thinking and methods to optimize this kind of vibrationisolating platform. The study obtained the following conclusions:

(1) The effect of platform damping ratio $\zeta$ on the displacement transfer rate is related to the jumping-down frequency $\Omega_{d}$ and external excitation frequency of the system. When the excitation frequency is in the high-frequency band that is higher than the jumpingdown frequency $\Omega_{d}$ of the platform, the smaller the damping ratio $\zeta$, the better. When the excitation frequency is in the low-frequency band that is lower than the jumping-down frequency $\Omega_{d}$ of the platform, the larger the damping ratio $\zeta$, the better.

(2) The effect of base displacement excitation amplitude on displacement transfer rate is related to the size of excitation amplitude $u_{0}$. The smaller the excitation amplitude, the lower the initial vibration isolation frequency, and the better the low-frequency vibration isolation effect, indicating that the vibration-isolating platform is suitable for small vibrations.

\section{Conflicts of Interest}

The authors declare that they have no conflicts of interest.

\section{Acknowledgments}

The authors would like to thank the financial supports of the Natural Science Foundation of Anhui Province (Grant nos. 1508085ME70 and 1708085ME127), of the National Natural Science Foundation of China (Grant no. 51575001), of Anhui University scientific research platform innovation team building projects (2016-2018), of Anhui Provincial Education Department Natural Science Foundation (Grant no. KJ2016A799), and of Wuhu Science and Technology Research Project (Grant no. 2014cxy 07).

\section{References}

[1] A. Carrella, M. J. Brennan, I. Kovacic, and T. P. Waters, "On the force transmissibility of a vibration isolator with quasi-zerostiffness," Journal of Sound and Vibration, vol. 322, no. 4-5, pp. 707-717, 2009.

[2] A. D. Shaw, S. A. Neild, and M. I. Friswell, "Relieving the effect of static load errors in nonlinear vibration isolation mounts through stiffness asymmetries," Journal of Sound and Vibration, vol. 339, pp. 84-98, 2015.

[3] W. S. P. Robertson, B. Cazzolato, and A. Zander, "Horizontal stability of a quasi-zero stiffness mechanism using inclined linear springs," Acoustics Australia, vol. 42, no. 1, pp. 8-13, 2014.

[4] Y. Zhang, B. Guo, and X. Meng, "Design and simulation for the single leg of the six degrees of freedom vibration isolator," Communications in Computer and Information Science, vol. 326, no. 1, pp. 269-276, 2012.

[5] D. Thayer and J. Vagners, "A look at the pole/zero structure of a stewart platform using special coordinate basis," in Proceedings of the American Control Conference, pp. 1165-1169, IEEE, Philadelphia, Pa, USA, June 1998.

[6] M. Guangfu, L. Yuhan, S. Yanchao, and M. Jingjing, "Active vibration isolation for Stewart platform using backstepping and NFTSM control," in Proceedings of the Navigation and Control Conference (CGNCC), pp. 1264-1269, IEEE, Nanjing, China, 2016.

[7] L.-Z. Ma, X.-Q. Yin, J. Xie, Q.-Z. Yang, and Z. Zhu, "Analysis of combined elastic damping device on multiple DOF parallel mechanism," Chinese Journal of Mechanical Engineering, vol. 40, no. 1, pp. 21-24, 2004.

[8] C. C. Yu, "Application of parallel mechanism in multi-DIM vibration absorbers," Applied Mechanics and Materials, vol. 532, pp. 297-300, 2014.

[9] X. Yin, Q. Yang, J. Xie, and P. Bao, "Vibration analysis of multi-DIM vibration absorber based on parallel mechanism," in Proceedings of the 2011 2nd International Conference on Mechanic Automation and Control Engineering, MACE 2011, pp. 7881-7884, Hohhot, China, July 2011.

[10] G. Gatti, I. Kovacic, and M. J. Brennan, "On the response of a harmonically excited two degree-of-freedom system consisting of a linear and a nonlinear quasi-zero stiffness oscillator," Journal of Sound and Vibration, vol. 329, no. 10, pp. 1823-1835, 2010.

[11] T. D. Le and K. K. Ahn, "A vibration isolation system in low frequency excitation region using negative stiffness structure for vehicle seat," Journal of Sound and Vibration, vol. 330, no. 26, pp. 6311-6335, 2011.

[12] J.-Y. Sun, H.-X. Hua, F. Xiao, X.-T. Liu, and X.-C. Huang, "Influence of nonlinear hysteretic damping on force transmissibility of a vibration isolation system," Journal of Vibration and Shock, vol. 33, no. 10, pp. 131-136, 2014.

[13] A. Carrella, M. J. Brennan, and T. P. Waters, "Static analysis of a passive vibration isolator with quasi-zero-stiffness characteristic," Journal of Sound and Vibration, vol. 301, no. 3-5, pp. 678689, 2007.

[14] J.-T. Chiu and C.-C. Fang, "Soft computing technologies in design of fuzzy controller for active vibration isolation systems," Journal of Marine Science and Technology (Taiwan), vol. 24, no. 3, pp. 519-529, 2016.

[15] A. Carrella, M. J. Brennan, and T. P. Waters, "Optimization of a quasi-zero-stiffness isolator," Journal of Mechanical Science and Technology, vol. 21, no. 6, pp. 946-949, 2007.

[16] D. L. Xu, Q. P. Yu, J. X. Zhou, and S. R. Bishop, "Theoretical and experimental analyses of a nonlinear magnetic vibration isolator with quasi-zero-stiffness characteristic," Journal of Sound and Vibration, vol. 332, no. 14, pp. 3377-3389, 2013.

[17] A. Murata, Y. Kume, and F. Hashimoto, "Application of catastrophe theory to forced vibration of a diaphragm air spring," Journal of Sound and Vibration, vol. 112, no. 1, pp. 31-44, 1987. 


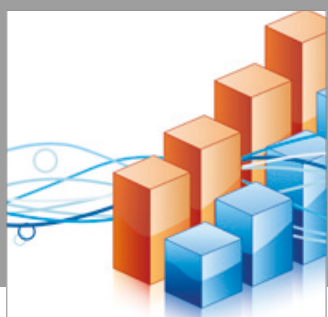

Advances in

Operations Research

vatersals

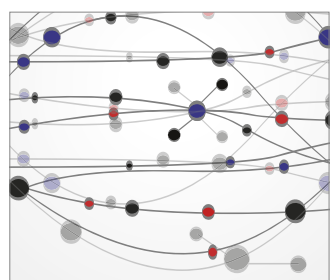

\section{The Scientific} World Journal
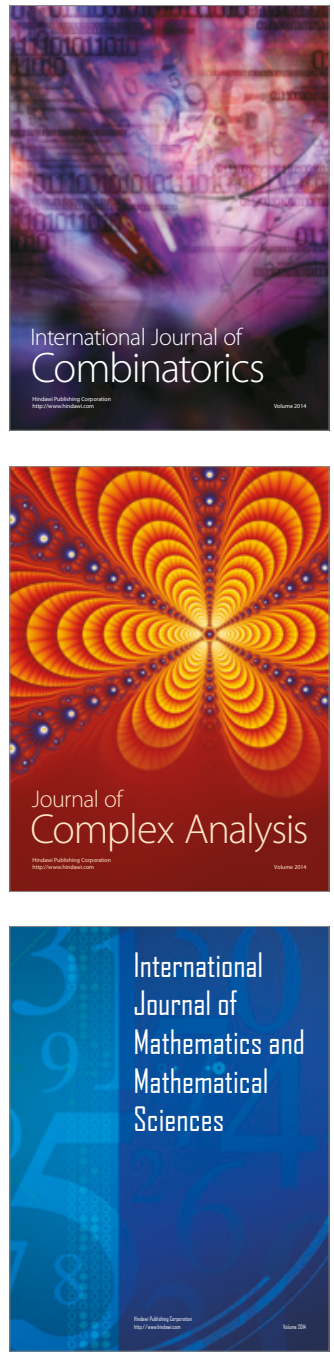
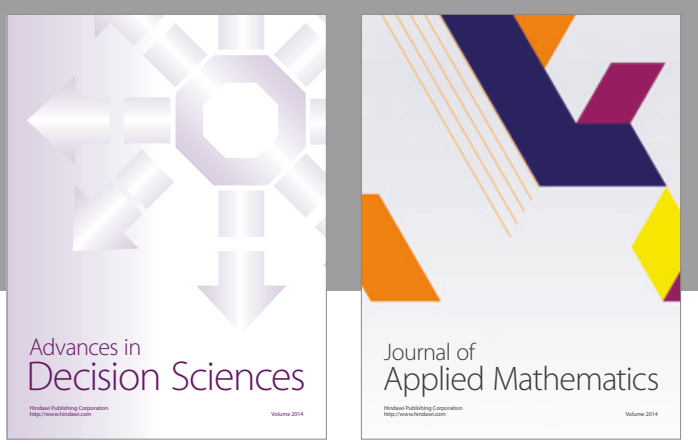

Algebra

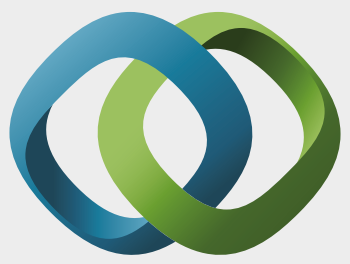

\section{Hindawi}

Submit your manuscripts at

https://www.hindawi.com
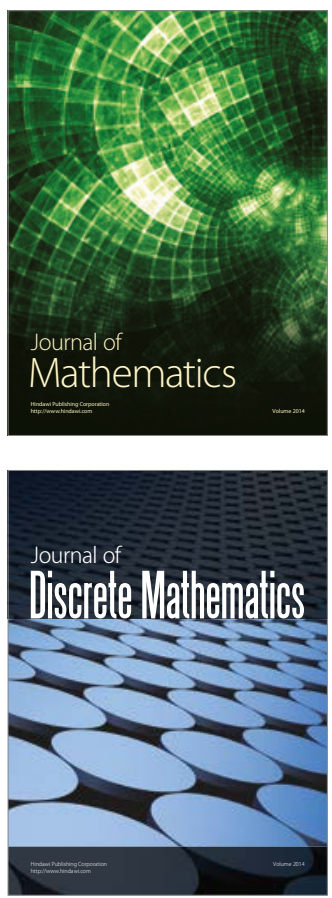

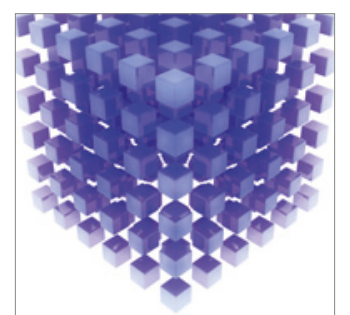

Mathematical Problems in Engineering
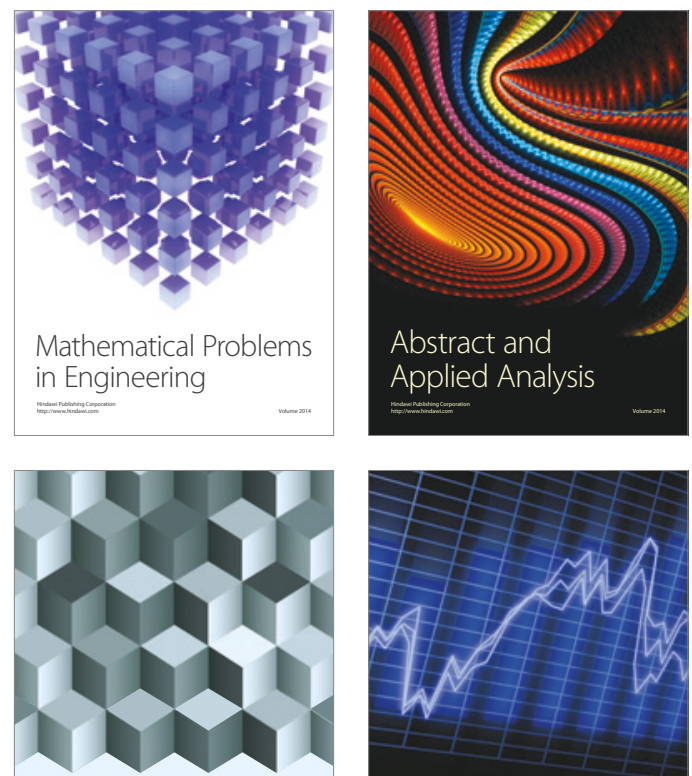

Journal of

Function Spaces

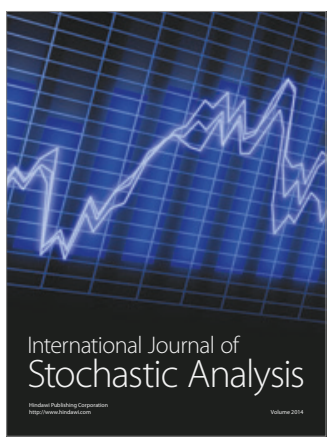

Probability and Statistics
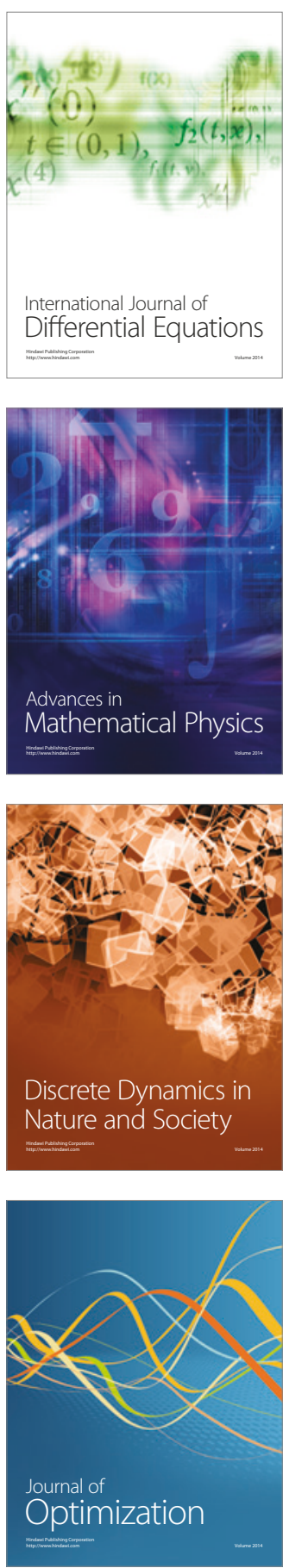